\title{
Comparative Analysis of Peak to Average Power Ratio Reduction in MIMO-OFDM
}

\author{
Apeksha Sonare ${ }^{1}$, Dinesh Bhoyar ${ }^{2}$ \\ PG Scholar, Electronics and Telecommunication Engineering, YCCE, Nagpur, India ${ }^{1}$ \\ Professor, Electronics and Telecommunication Engineering, YCCE, Nagpur, India ${ }^{2}$
}

\begin{abstract}
Multiple input multiple output (MIMO) technique, one of the multicarrier communication systems has become promising characteristics. MIMO with orthogonal frequency division multiplexing (OFDM) constitutes the MIMO-OFDM. This MIMO-OFDM systems faces the problem of non-constant large peaks results in peak-to-average power ratio which is major drawback of multicarrier systems due to which there is reduction in spectral efficiency and increased in system complexity. This paper presents analysis and comparison two PAPR reduction techniques. Selection mapping (SLM) for different route numbers which is one of efficient technique to reduce PAPR when the number of subcarriers is large. Simulation shows that PAPR problem reduces as route number increases. Partial transmit sequence (PTS) technique presents low complexity by applying new phase sequence, Simulation results shows PAPR reduction by PTS method.
\end{abstract}

Keywords: MIMO (Multiple input multiple output), OFDM (Orthogonal frequency division multiplexing), PAPR (Peak to average power ratio), SLM (Selection mapping), PTS (Partial transmit sequence).

\section{INTRODUCTION}

A Multiple input multiple output (MIMO) with orthogonal frequency division multiplexing (OFDM) has recent in trends and attracted many researchers to increase the spectral efficiency, low complexity and degradation of bit error rate (BER) performance. MIMO with OFDM is most efficient multicarrier communication system due its advantages such as reduction in complexity, increased in efficiency, reduction in linear distortion etc. With this it has become very promising in the wireless communication systems. MIMO-OFDM uses the principle of frequency division multiplexing in controlled manner allowing to improve the efficiency of the system. The basic principle of MIMO-OFDM is to split high data rate streams into number of lower data rate streams over number of subcarriers which overlapped with each other. MIMO-OFDM faces several challenges. The main challenges are peak-to-average power ratio due to nonlinearity of amplifier, Phase noise problems of oscillators, ISI due to multipath use guard interval and many more. In this paper we focused on issue of peak to average power ratio reduction in MIMO-OFDM systems. The transmit signal in the MIMO-OFDM may have high peak values in time domain since many subcarrier components are added by via IFFT operation, therefore MIMO-OFDM are known to have PAPR (Peak-to-average power ratio) compared to single carrier communication systems. When the transmit signal in MIMO-OFDM pass through the device that is nonlinear device may be high power amplifier this results and causes the out of band distortion which effects the signals in inband distortion, adjacent bands. So due to this a large input back-off power is required to further proceed the process of operation in linear region of high power amplifier (HPA).Such high power amplifiers (HPA) have large dynamic range and it increases the overall costs of the system. By the PAPR reduction the overall cost of the system reduce as well as the complexity of various components withheld in the MMO-OFDM system.

To reduce or solve the problem of PAPR different schemes are developed till now such clipping technique in this the large peaks of the signals are clipped that is clip the signal around the peaks but with the increased in distortion. Under this technique filtering, block scaling, Fourier projection, Peak cancellation involves. Despite of this coding techniques are also used to reduce the PAPR of the MIMO-OFDM system such as Golay complementary sequences, Hadamard, Reed Muller codes, but by all this approaches there is reduction in the PAPR value by increased in system complexity and low bandwidth efficiency. To handle the time variation of high power amplifier (non-linear) by modifying constellation extension. For the reduction of PAPR transform schemes are also used such as DFT-spreading, DHT (Discrete Hilbert Transform).

Widely used PAPR reduction techniques are signal scrambling techniques. Probabilistic scrambling techniques in which the input data block is scrambled and the sequence having the low PAPR is transmitted, techniques includes selection mapping (SLM) and Partial Transmit Sequence (PTS), Tone injection, Tone reservation techniques. These techniques are those who does not suffer from out-of-band distortion or out-of-band power so as spectral efficiency decreases and there is increase in complexity as the number of subcarriers increases. Among all these techniques, this 
paper reviews on the selection mapping technique (SLM) and Partial transmit sequence technique (PTS), and the comparison of both the techniques that which the most efficient one for PAPR reduction.

\section{SYSTEM MODEL}

Subcarriers ' $\mathrm{N}$ ' are taken for transmission of OFDM and also the ' $\mathrm{N}$ ' be length of IDFT. All ' $\mathrm{N}$ ' subcarriers are necessary to be active and for each transmitters that is ' $\mathrm{m}$ ' parallel OFDM transmitters, $\mathrm{N}$ distinct complex value which further converted from frequency to time domain by inverse Fast Fourier Transform (IFFT). The input signal given by-

$$
x_{m, k}=\operatorname{IFFT}\left(X_{m, k}\right)=\frac{1}{\sqrt{N}} \sum_{k=0}^{N-1} X_{m, n} \exp (j 2 \pi n k / N)
$$

Where $\mathrm{m}=1,2 \ldots . \mathrm{M}$ and $\mathrm{k}=1,2 \ldots \mathrm{N}-1$, are considered as variance for same constellation. The samples in the time domain () are complex Gaussian distributed results high PAPR so as the carriers are independent statistically.

$$
P A P R_{m} \equiv \frac{\max _{k}\left|x_{m, k}\right|^{2}}{E\left\{\left|x_{m, k}\right|^{2}\right\}} \equiv \frac{\max _{k}\left|x_{m, k}\right|^{2}}{\sigma_{X}^{2}}
$$

So in order to avoid the out-of-band radiation or out-of-band power for different ' $M$ ' route numbers the PAPR value of the system should be less or small, But in MIMO-OFDM there are $\mathrm{M} * \mathrm{~N}$ samples in the time domain are present whereas ' $\mathrm{N}$ ' time domain samples in single OFDM system. Best efficiency or performance of the system can be evaluated with the worst or minimum PAPR. For PAPR value the CCDF of MIMO-OFDM is given by-

$$
\begin{aligned}
& x_{m}(t)=\frac{1}{\sqrt{N}} \sum_{n=0}^{N-1} X_{n} P_{m, n} \cdot e^{j 2 \pi n \Delta f t} \\
& \operatorname{Pr}\left\{P A P R_{\text {MIMO }} \succ P A P R_{o}\right\}=1-\left(1-e^{-P A P R_{o}}\right)^{M N}
\end{aligned}
$$

\section{III.METHODOLOGY}

There are different versions for the PAPR reduction in MIMO-OFDM that are previously studied. Some modification in SLM (selection mapping) and PTS (partial transmit sequence) are made in this review and also the comparison of both technique that which one is more efficient for the PAPR reduction in point-to-point transmission for MIMOOFDM systems.

\section{A. SELECTION MAPPING (SLM)}

The main idea behind the selection mapping (SLM) is to generate the set consist of sufficiently different data blocks by the transmitter in which all the data blocks represents the same information as that of the original data block and then select the one favourable which have the least PAPR value for the transmission. The Block diagram of SLM further showing the basic principles of selection mapping-

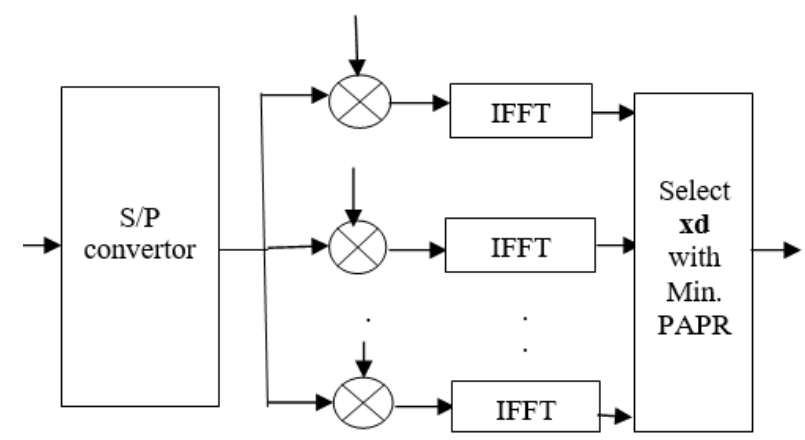

Fig 1Basic Principles of Selection Mapping 
The data blocks in each involvement have the ' $\mathrm{N}$ ' modulated symbols that are copies to form several copies of ' $\mathrm{M}$ ' which carries the information same as the original one. Then this data blocks are multiplied by M different phase sequences which are of length $\mathrm{N}$ resulting in different modified $\mathrm{M}$ data blocks,

$$
P_{m}=\left[P_{m, 0}, P_{m, 1, \ldots,} P_{m, N-1}\right]^{T}
$$

Here $\mathrm{m}=1,2, \ldots \mathrm{M}$.

Let us consider symbol that is modified data blocks in branch $\mathrm{m}$ can be expressed as-

$$
S_{m}=\left[X_{0} P_{m, 0}, X_{1} P_{m, 1, \ldots . .} X_{N-1} P_{m, N-1}\right]^{T}
$$

Where $\mathrm{m}=1,2, \ldots . \mathrm{M}$ for phase sequence.

After the conversion from frequency domain to time domain of OFDM symbols by performing the IFFT operations the whole process can be expressed by-

$$
x_{m}(t)=\frac{1}{\sqrt{N}} \sum_{n=0}^{N-1} X_{n} P_{m, n} \cdot e^{j 2 \pi n \Delta f t}
$$

As a result, one which has or posses the minimum PAPR is selected for transmission. Mathematically it can be given by-

$$
x_{d}=\arg \min _{1 \leq m \leq M}\left(\operatorname{PAPR}\left(x_{m}\right)\right)
$$

Here argmin () represents the judgment condition for minimum PAPR.

But it has been found that this condition is very probabilistic and time consuming requires large number of IFFT operations although PAPR value is minimized. So it must be necessary to further implementation of the other techniques for the reduction of PAPR in MIMO-OFDM systems.

\section{B. PARTIAL TRANSMIT SEQUENCE (PTS)}

Partial transmit sequence (PTS) is one of the most promising technique for the reduction of PAPR as its performance is without occurring distortion.In this technique the input is subdivided into sub-blocks and that are then multiplied by weighting phase sequences for multiple sequence which transmit (Partial transmit sequence) out of this the one which carries the minimum PAPR is selected for transmission, So to find the optimum one search is made over all combination of phase factors and this results increased in complexity of the system. In order to reduce the computational complexity of many proposed schemes had suggested for reducing the sub-blocks.

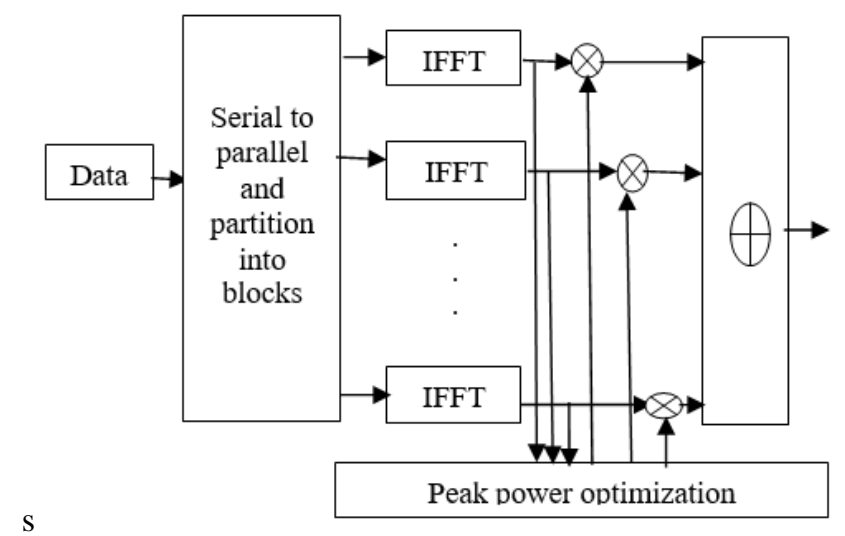

Fig 2 Block Diagram of Partial Transmit Sequence

The data vector is divided into $\mathrm{V}$ disjoints sets and using that same no. of carriers from $\mathrm{V}$ group, each sub-blocks having the same size of length $\mathrm{N}$ then the sub-block vector is given by- 


$$
\hat{X}=\sum_{v=1}^{V} b_{v} X_{v}
$$

Where

$b_{v}=e^{j \varphi v}\left(\varphi_{v} \in[0,2 \pi]\right)$

Here $\mathrm{v}=1,2, \ldots \mathrm{V}$, is the weighting factor that is used for the purpose of phase rotation.

Where $\operatorname{argmin}($.$) represents the judgement condition shows that the PAPR value is minimized.The additional cost in$ this technique we have to pay is extra v-1 times the IFFT operations. In the conventional approach of PTS technique, $b$ phase factor is send to the receiver as the side band information so that the redundancy bits for $\mathrm{V}$ number of sub-blocks and $\mathrm{W}$ for possible variation of phase which causes the more burden on MIMO-OFDM system and hence effects the complexity.

\section{IV.SIMULATION RESULTS}

In the simulation results the outcomes of the proposed schemes are shown. In the selection mapping technique (SLM) the route number $\mathrm{M}$ is affected so also the subcarriers $\mathrm{N}$. The simulations results for SLM technique is observed for different route numbers with respect to subcarriers N. It has been found that the PAPR value has been decreased for increased the route numbers $\mathrm{M}$, but the complexity increases. From the results it has been seen the algorithm of technique executes for 10000 times oversampling factor is 8 and QPSK mapping is taken under consideration as modulation scheme. Up till now it has been observed the PAPR reduction for $\mathrm{M}=64$ route numbers but the proposed scheme shows better results for increased in route number $M=128$. With increase in route number from $M=2 M=4 M=8$ $\mathrm{M}=16 \mathrm{M}=32 \mathrm{M}=64$ and $\mathrm{M}=128$ for $\mathrm{N}$ subcarriers there is decreased in PAPR value Than original OFDM system. Increase in route number improves the performance of PAPR reduction so the probability of high PAPR is reduced. If to $1 \%$ the probability is set, then for different values of route numbers $\mathrm{M}$ for CCDF curves compared, it has been fond PAPR reduced smaller for $M=2$ than unmodified $M=1$. Moreover from comparison of route number $M=8$ and $M=16$ the PAPR performance less than $0.5 \mathrm{~dB}$, this proves that there is no linear growth for further increase in value of $\mathrm{M}$. Thus in practical it consider for $\mathrm{M}=8$, this not only improves the performance but also avoid the complexity of system within the limited available resource.

$$
\hat{x}=\operatorname{IFFT}(\hat{X})=\sum_{v=1}^{V} b_{v} \operatorname{IFFT}\left(X_{v}\right)=\sum_{v=1}^{V} b_{v} \cdot x_{v}
$$

The best suitable combination of factor which makes the Result optimum is given by-

$$
b=\left[b_{1}, b_{2}, \ldots . . b_{v}\right]=\arg \min _{\left(b_{1}, b_{2}, \ldots b_{v}\right)}\left(\max _{1 \leq n \leq N}\left|\sum_{v=1}^{V} b_{v} x_{v}\right|^{2}\right)
$$

Fig.3 shows the simulation results of selection mapping technique (SLM) from that it can be analyze that as the route number $\mathrm{M}$ increases and subcarriers $\mathrm{N}$ so there has been decrease in the value of PAPR improving the statistics of multicarrier signaling also the performance of system. This technique results in PAPR reduction for different values of $\mathrm{M}$, but it does not fully overcome the problem of system complexity.

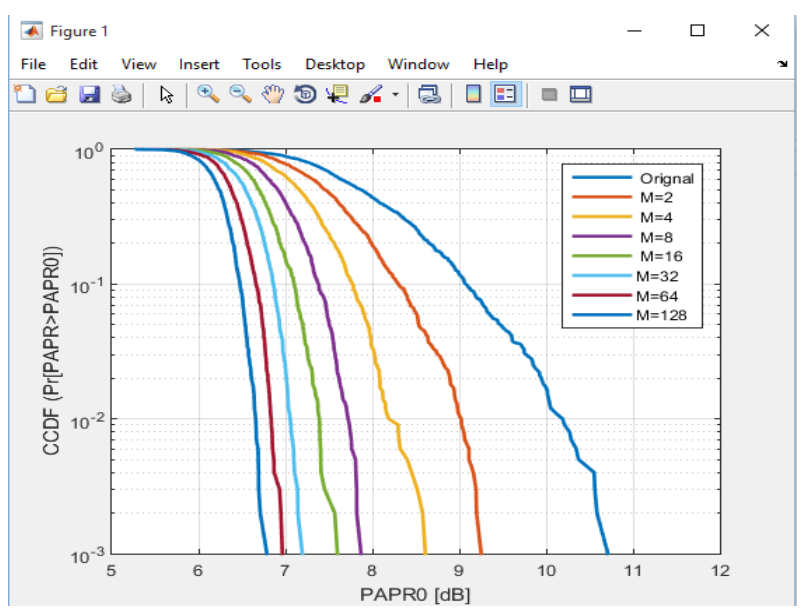

Fig 3. Comparison of PAPR Reduction Schemes for Different Values of Route numbers M 
Fig.4 shows the simulations results for the partial transmit technique (PTS), Simulation results evaluates with algorithm of PTS for different sub-blocks V. QPSK is assumed as modulation scheme, subcarriers $\mathrm{N}=256$ and $\mathrm{v}=0,2,3,4$ respectively. PAPR performance gets affected by different values of $\mathrm{M}$ that is collection range of weighting factor $\mathrm{W}$. Simulation parameters $\mathrm{N}=128$ oversampling factor takes $\mathrm{L}=8$ enhance sub-blocks $\mathrm{v}=4$. The PAPR performance CCDF curve for fixed sub-block and varying weighting factor $\mathrm{W}$, it has been found that $\mathrm{W}=4$ than $\mathrm{W}=2$ the $1 \%$ PAPR is about $7.5 \mathrm{~dB}$. Therefore for larger value of $\mathrm{W}$ there has been observed better performance of PAPR.

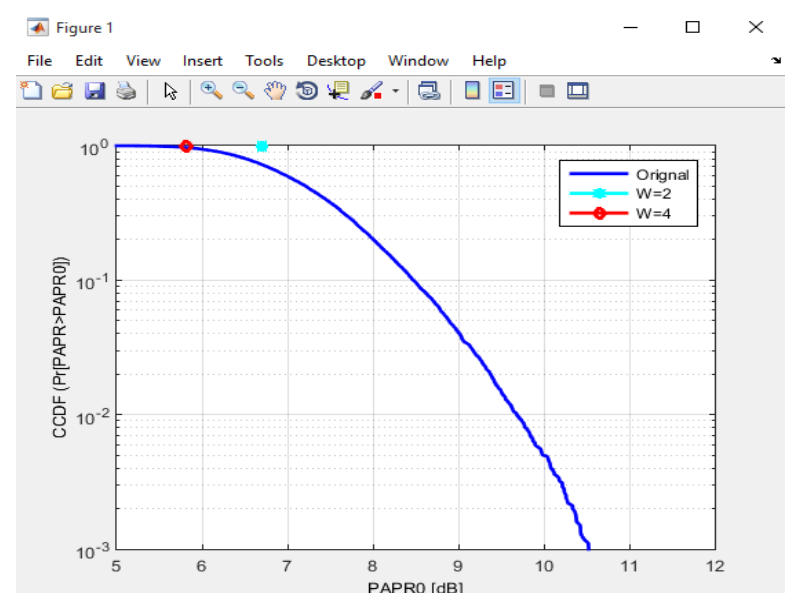

Fig 4. Comparison of PAPR Reduction Performance For Different Values of W

\section{FUTURE SCOPE}

The future scope is that, we can fully explore the advantages of combination between proposed PAPR reduction schemes and outstanding properties of MIMO-OFDM system, suchas studying the PAPR reduction technology of MIMO-OFDM system, combine with space-time codes.

\section{CONCLUSION}

In this project, a SLM and PTS method has been presented for PAPR reduction in MIMO-OFDM. The PAPR reduction increases as the number of SLM vectors is increased. With increasing route number, the PAPR is further improved. The problem of PAPR in OFDM and MIMO-OFDM is an ongoing issue. The combined technique can be applied to varying factors and performance of SLM and PTS will be compared. Various algorithms can be devised for decreasing the complexity of the MIMO-OFDM systems.

\section{REFERENCES}

[1] Young Kyun Kim, Ramjee Prasad, "What is 4G," in 4G Roadmap and Emerging Communication Technologies, Artech House, 2006, pp. 1213.

[2] University of Alberta, "Home page - High capacity digital communications laboratory," 2007. [Online]. Available: http://www.ece.ualberta. $\mathrm{ca} / \sim \mathrm{HCDC} /$ mimohistory.html [Accessed: July. 12, 2009].

[3] Mohinder Jankiraman, "Multicarrier techniques," in Space-time codes and MIMO systems, Artech House, 2004, pp. 15.

[4] Shinsuke Hara, Ramjee, "Principle and history of MCM/OFDM," in Multicarrier techniques for 4G mobile communication, Artech House, 2003, pp. 39

[5] E. Telatar, "Capacity of multi-antenna Gaussian channels,” European Transactions on Telecommunications, vol. 10, no 3, Dec 1999, pp. 585595.

[6] Foschini G J, Gans M J, "On limits of wireless communication in a fading environment when using multiple antennas," Wireless Personal Communication, vol. 6, no 3, Sep 1998, pp. 311-335.

[7] Mohinder Jankiraman, "MIMO system capacity," in Space-time codes and MIMO systems, Artech House, 2004, pp. 23.

[8] KUANG Yu-jun, TENG Yong, "A new symbol synchronization scheme for cyclic prefix based systems," The Journal of China Universities of Posts and Telecommunications, vol. 10, no 3, 2003, pp. 5-8.

[9] Lei Xia, Xiao Yue, et al., "Analysis of the performance of partial transmit sequences with different sub-block partitions," IEEE International Conference on Communication, Circuits and Systems Proceedings, ICCCAS June 2006, vol. 2, pp. 857-858. 